\title{
Influence of Magnetic Helicity in MHD
}

\author{
Simon Candelaresi, Fabio Del Sordo and Axel Brandenburg \\ NORDITA, AlbaNova University Center, Roslagstullsbacken 23, SE-10691 Stockholm, Sweden \\ and \\ Department of Astronomy, Stockholm University, SE 10691 Stockholm, Sweden
}

\begin{abstract}
Observations have shown that the Sun's magnetic field has helical structures. The helicity content in magnetic field configurations is a crucial constraint on the dynamical evolution of the system. Since helicity is connected with the number of links we investigate configurations with interlocked magnetic flux rings and one with unlinked rings. It turns out that it is not the linking of the tubes which affects the magnetic field decay, but the content of magnetic helicity.
\end{abstract}

Keywords. Sun: magnetic fields

Magnetograms of the Sun's surface have shown (Pevstov et al. 1995) that the field lines in the active regions are twisted. From observations of the chromosphere and the corona it was conjectured (Leka et al. 1996) that the magnetic field in sunspots gets twisted before it emerges. Extrapolation of the three-dimensional structure of magnetic field lines have shown twist in the field (Gibson et al. 2002). Since twisting is connected to helicity we can say that the Sun's magnetic field shows helical patches.

For two flux tubes which are not twisted and do not intersect each other the magnetic helicity is related to the number of mutual linking via the formula (Moffatt 1969)

$$
H=\int_{V} \mathbf{A} \cdot \mathbf{B} \mathrm{d} V=2 n \phi_{1} \phi_{2}
$$

where $H$ is the magnetic helicity, $\mathbf{B}=\nabla \times \mathbf{A}$ is the magnetic field in terms of the vector potential $\mathbf{A}, \phi_{1}$ and $\phi_{2}$ are the magnetic fluxes through the tubes and $n$ is the linking number. Since $H$ is a conserved quantity in ideal MHD the linking number is also conserved.

In ideal MHD and in presence of magnetic helicity the spectral magnetic energy is bounded from below through the realizability condition (Moffatt 1969)

$$
M(k) \geqslant k|H(k)| / 2 \mu_{0} \quad \text { with } \quad \int M(k) \mathrm{d} k=\left\langle\mathbf{B}^{2}\right\rangle / 2 \mu_{0}, \quad \int H(k) \mathrm{d} k=\langle\mathbf{A} \cdot \mathbf{B}\rangle
$$

and the magnetic permeability $\mu_{0}$ and where $\langle$.$\rangle denotes volume integrals. It is the aim$ of our work (Del Sordo et al. 2010) to study the dynamical evolution of a system with interlocked flux rings with and without helicity.

The setups under consideration consist of three flux tubes with constant magnetic flux. For two configurations the rings are interlocked while for one they are separated. In one of the interlocked configurations we change the direction of the flux such that the magnetic helicity becomes zero (Fig. 1). The inner ring has a radius which is 1.2 times larges then for the outer rings. We solve the full resistive MHD equations for an isothermal and incompressible medium.

For the interlocked configuration with finite magnetic helicity the magnetic energy decays with a power law of $t^{-1 / 2}$, while both non-helical configurations decay much faster with a power law of $t^{-3 / 2}$ (Fig. 2). 

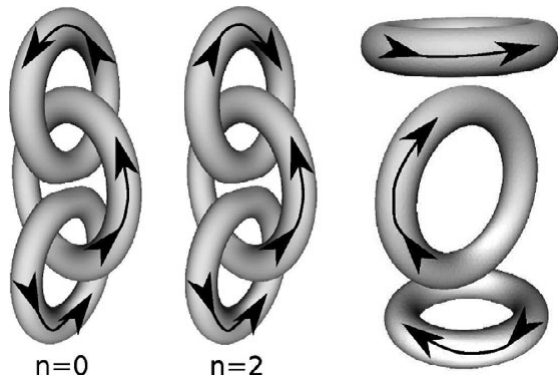

Figure 1. The initial triple ring configuration. Interlocked rings with $n=0$ (left) and $n=2$ (center) and non interlocked configuration with $n=0$ (right). The arrows indicate the direction of the magnetic field.
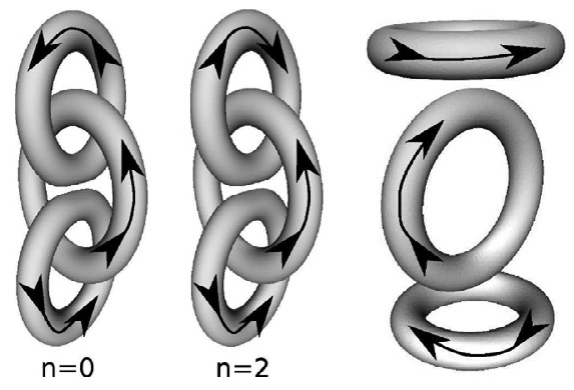

Figure 2. Magnetic energy decay normalized for the initial time for linking number 2 (solid line), 0 (dashed line) and the non-interlocked case (dotted line).

One can see (Fig. 3) that the linked structure is conserved for the finite helicity case. For even later times the linking gets transformed into twisting.
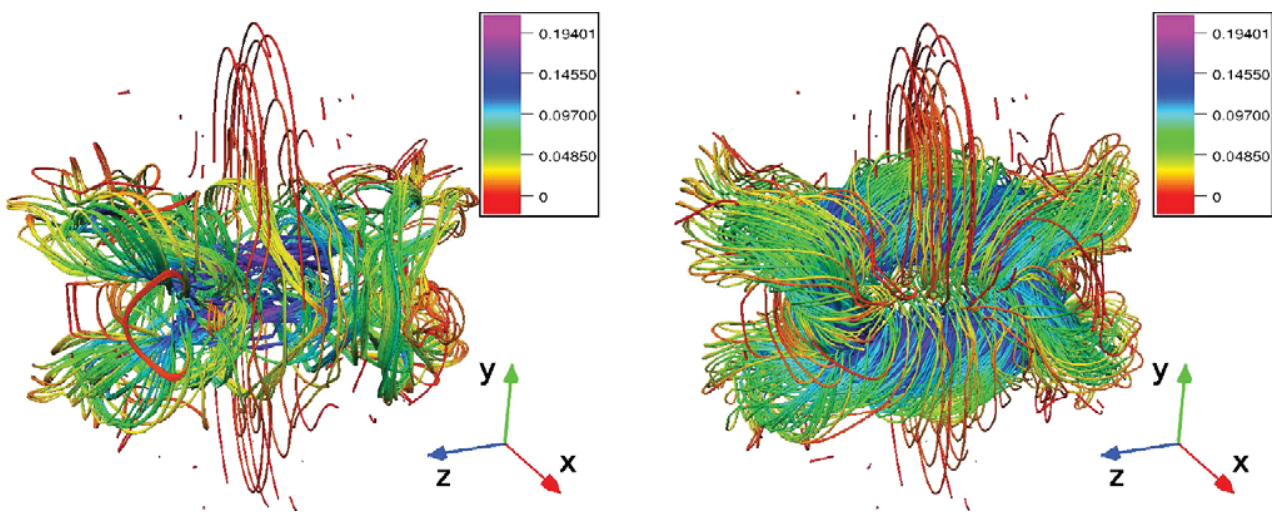

Figure 3. Magnetic field configuration after 4 Alfvén times for the case of linking number zero (left) and finite linking number (right). The colors represent the magnitude of the magnetic field.

Due to the realizability condition the decay of magnetic energy is slowed down by the magnetic helicity which decays slowly. Since helicity is decaying slowly and is almost conserved also the number of linkings is almost conserved. This is why the linking is transformed into twisting of the fields which then contributes to the helicity. The test run with non-interlocked rings shows that it is the helicity content of the system and not the actual number of linkings which affects the decay.

\section{References}

Moffatt, H. K., J. Fluid Mech. 35, 117 (1969).

Pevtsov, A. A., Canfield, R. C. \& Metcalf, T. R. 1995, ApJ, 440, L109

Leka, K. D., Canfield, R. C., McClymont, A. N., van Driel-Gesztelyi, L., ApJ 462, 5471996

Gibson, S. E., Fletcher, L., Del Zanna, G., et al., ApJ 574, 10212002

Del Sordo, F., Candelaresi, S., Brandenburg, A., Phys. Rev. E 81, 0364012010 